\title{
Principles of Space-Time Array Processing for Ultrawide-Band Impulse Radar and Radio Communications
}

\author{
Malek G. M. Hussain, Senior Member, IEEE
}

\begin{abstract}
Over the last decade of the 20th century, the emerging ultrawide-band (UWB) impulse technology has found numerous applications in the commercial as well as the military sectors. The rapid technological advances have made it possible to implement (cost-effective, short-range) impulse radar and impulse-radio communication and localization systems. Array beamforming and space-time processing techniques promise further advancement in the operational capabilities of impulse radar and impulse-radio communications to achieve long-range coverage, high capacity, and interference-free quality of reception. In this paper, we introduce a realistic signal model for UWB impulse waveforms and develop the principles of space-time array processing based on the signal model. A space-time resolution function (STRF), a space-frequency distribution function (SFDF), and a monopulse-tracking signal are derived for impulse waveforms received by a self-steering array beamforming system. The directivity peak-power pattern and energy pattern of the beamformer are also derived. Computer plots of the STRF, SFDF, and the beam patterns are obtained too. The directivity beam patterns of impulse waveforms are sidelobe-free and, therefore, there is no need for sidelobe suppression via amplitude weighting of the array elements. Also, the resolution angle for the beam patterns is derived as a decreasing function of array size and frequency bandwidth. Electronic beamsteering based on slope processing of monopulse waveforms is described too.
\end{abstract}

Index Terms-Adaptive array antenna, array beamforming, impulse radar, impulse radio, space-time processing, ultrawide-band (UWB) technology.

\section{INTRODUCTION}

$\mathbf{T}$ $\checkmark$ HE CONCEPT of adaptive array antenna was introduced almost four decades ago to advance the performance of military radar (and possibly radio communications) operating in a dense signal environment, where active interference and jamming can be present and pose a major threat [1]-[8]. Adaptive array antenna electronically points a main beam toward a desired signal source without a priori knowledge of the source's angular position and, at the same time, suppresses interference and noise arriving from other look directions. The operation of such an antenna system is controlled by an adaptive signal processing algorithm that is designed to achieve specific performance criteria for a desired signal. The recent

Manuscript received June 29, 2000; revised September 5, 2001

The author is with the Department of Electrical Engineering, Kuwait University, Al-Safat 13060, Kuwait (e-mail: malek@kuc01.kuniv.edu.kw).

Publisher Item Identifier S 0018-9545(02)02513-6. advances in solid-state electronics and digital technologies have made it possible and cost effective to implement adaptive-array concepts in commercial radio communication systems for improving overall system capacity, coverage, and quality of reception [9], [10]. Space-time array signal processing for the applications of conventional radar and wireless communications remains an active area of research (a comprehensive list of references is given in [10]).

The concept of space-time array processing is also applicable to the emerging technology of ultrawide-band (UWB), impulse (or carrier-free), radar, and radio communications [11]-[24]. To date, UWB impulse technology demands much research work to achieve enhanced operational capabilities and cost-effective system production for wide commercial applications. Theoretical research in the area of signal modeling and signal processing is essential for the design, performance analysis, and development of practical UWB systems.

In this paper, the principle of space-time impulse-array processing is presented based on a realistic signal model for UWB impulse waveforms. Correlation processing (in time domain) of impulse waveforms yields fine temporal resolution that is attractive for isolating and eliminating multipath reception and cochannel interference. On the other hand, array beamforming or simply space processing based on impulse waveforms results in a directivity beam pattern having a narrow mainlobe and practically no sidelobes. Such beam pattern yields high angular resolution and neutralizes the need for amplitude weighting at each array element.

The analytical models in time and frequency domain of UWB impulse waveforms are introduced in Section II. The principle of a self-steering array beamforming system for impulse waveforms is described in Section III. Also, space-time resolution function (STRF) and space-frequency distribution function (SFDF) are derived and plotted. The STRF and the SFDF result in a directivity energy pattern that allows for a tradeoff between frequency bandwidth and array size for a small resolution angle. Such a tradeoff is of interest in practice since the attenuation due to the media in which the signal propagates is an increasing function of frequency. Directivity patterns of impulse waveforms are derived and plotted in Section III too. The mechanism of electronic beamsteering based on computer processing of monopulse-tracking signals is presented in Section IV. Derivation and plots of the monopulse signal are also presented. Conclusions are given in Section V. 


\section{Signal Model FOR IMPUlSe TRANSMisSiON}

\section{A. Impulse-Based Spread-Spectrum Signals}

The conventional technology of radar and radio communications is based on the use of a sinusoidal carrier as a modulation waveform, which, on the theoretical side, allows for the mathematically convenient complex representation of bandlimited signals. For the emerging technology of impulse radar and impulse-radio communications, the carrier signal (or the modulation waveform) is a uniform train of UWB pulses represented as

$$
s(t)=\sum_{n=-\infty}^{+\infty} \Omega\left(t-n T_{r}\right)
$$

where $T_{r}$ is the pulse repetition interval (PRI), and $\Omega(t)$ is the pulse-shaping waveform. The energy spectral density of $\Omega(t)$ must be free from a dc component since, according to antenna principles, the presence of a dc component prevents the direct radiation of electromagnetic baseband pulses without an appropriate modulation of a sinusoidal carrier. A physically realizable signal model for $\Omega(t)$ is presented in Section II-B. Conventional digital communication systems transmit data via one of the following modulation techniques: amplitude-shift keying (ASK), frequency-shift keying (FSK), and phase-shift keying (PSK). Spread-spectrum (SS) modulation techniques, i.e., direct sequence (DS), frequency hopping (FH), or a hybrid DS-FH, are also employed to achieve multiple-access capabilities and secure communications.

In the applications of impulse-radio technology, information transmission is achieved by encoding the impulse-based carrier signal given in (1). For example, digital-impulse-radio communication systems transmit binary data symbols $(0$ and 1) by coded sequences of $N_{s}$ UWB pulses. The coding scheme can either be applied to the PRI or directly to the amplitude of the individual pulses depending on the system complexity. The coding (or staggering) of the PRI is referred to as time-hopping (TH) SS modulation [20], [21]. The amplitude coding scheme is the same as the well known direct-sequence SS modulation. Also, it is possible to implement a hybrid DS-TH coding scheme, which yields improved system capacity and security but on the account of a more costly and complex system. The hybrid DS-TH would be analogous to the known hybrid direct-sequence and frequency-hopping (DS-FH) SS technique. In general, the signal model for a DS-TH waveform representing a binary data symbol $D_{0}=0$ or $D_{1}=1$ is written as follows:

$$
s_{D S T H}^{(j)}(t)=\sum_{n=0}^{N_{s}-1} q_{n}^{(j)} \Omega\left(t-n T_{r}-c_{n}^{(j)} T_{c}-T_{d} D_{i}\right) .
$$

In (2), the index $i=\{0,1\}$, the index $j$ denotes the $j$ th user in a multiple-access system, $\left\{q_{n}^{(j)}\right\}$ is a binary pseudorandom (PN) code of length $N_{s}$ and elements +1 and $-1,\left\{c_{n}^{(j)}\right\}$ is also a PN code referred to as the time-hopping code with integer elements in the range $0 \leq c_{n}^{(j)} \leq N_{h}, T_{c} \ll T_{r}$ is the chip duration of the TH code, and $T_{d} \ll T_{r}$ is the data-modulation time shift that conveys to the receiver information regarding the symbol $D_{0}$ or $D_{1}$. The symbol duration is $T_{s}=N_{s} T_{r}$ and the binary symbol rate is $R_{s}=1 / T_{s}=1 / N_{s} T_{r}$. The maximum time-hopping interval is $N_{h} T_{c} \leq T_{r}$. In this case, information transmission is accomplished via pulse-position modulation (PPM) while multiple-access capability is achieved by DS-TH SS modulation. Note that the hybrid DS-TH modulation is not a requirement since any one SS modulation method can be sufficient for all practical purposes.

The signal structure given in (2), with $T_{d}=0$ also represents an SS radar signal having a low probability of intercept and high resistance against jamming. This "stealthy" feature of impulse waveforms is one of the potential advantages of UWB impulseradio technology.

\section{B. Representation of Impulse Waveforms}

Based on experimentation with UWB impulse radiators and sensors [22], the time variation of the pulse-shaping waveform $\Omega(t)$ given in (1), may analytically be represented by the time function

$$
\begin{aligned}
\Omega(t)= & \frac{E_{0}}{1-\alpha}\left(\exp \left\{-4 \pi\left[\left(t-t_{\circ}\right) / \Delta T\right]^{2}\right\}\right. \\
& \left.-\alpha \exp \left\{-4 \pi\left[\alpha\left(t-t_{\circ}\right) / \Delta T\right]^{2}\right\}\right), \quad \alpha \neq 1 .
\end{aligned}
$$

In (3), $E_{\mathrm{o}}$ is the peak amplitude at the time $t=t_{0}, \Delta T$ is a nominal duration, and $\alpha$ is a scaling parameter. The function $\Omega(t)$ is referred to as the generalized Gaussian pulse (GGP). The time variation of the normalized GGP signal $\Omega(t) / E_{0}$ is plotted in Fig. 1(a) as a function of relative time $t / \Delta T$ for $t_{0}=0$ and $\alpha=0$ (dotted line), 0.75 (dashed line), 1.5 (dashed-dotted line), and 3 (solid line). The GGP signal has an even symmetry about its peak amplitude at $t_{\circ} / \Delta T$ and it is characterized by a positive mainlobe and two equal time sidelobes having purely negative values, which yields the average value $\int_{-\infty}^{+\infty} \Omega(t) d t=$ 0 . Hence, the GGP is a baseband pulse with no dc component in its energy density spectrum, as we shall demonstrate shortly. According to Fig. 1(a), the duration of the mainlobe and the magnitude of the time sidelobes are decreasing functions of the scaling parameter $\alpha$. For $\alpha=0$, the negative time sidelobes vanish and $\Omega(t)$ becomes an ideal Gaussian pulse, as shown in Fig. 1(a) by the dotted line.

The autocorrelation function $\Upsilon(t)$ of the GGP signal is expressed as follows:

$$
\begin{aligned}
\Upsilon(t) & =\int_{-\infty}^{+\infty} \Omega(\lambda) \Omega(\lambda+t) d \lambda \\
& =E_{\mathrm{o}}^{2} \Delta T \sum_{k=0}^{2} I_{k} \exp \left\{-a_{k}\left[\left(t-t_{\circ}\right) / \Delta T\right]^{2}\right\}
\end{aligned}
$$

where

$$
\begin{aligned}
& I_{0}=1 / \sqrt{8}(1-\alpha)^{2}, \quad a_{0}=2 \pi \\
& I_{1}=\alpha / \sqrt{8}(1-\alpha)^{2}, \quad a_{1}=2 \pi \alpha^{2} \\
& I_{2}=-\alpha /(1-\alpha)^{2}\left(1+\alpha^{2}\right)^{1 / 2}, \quad a_{2}=4 \pi \alpha^{2} /\left(1+\alpha^{2}\right) .
\end{aligned}
$$

The energy $\mathcal{E}$ of the GGP signal $\Omega(t)$ is the peak value of the autocorrelation function $\Upsilon(t)$ at $t=t_{0}$

$$
\mathcal{E}=\Upsilon\left(t_{\circ}\right)=\frac{E_{\circ}^{2} \Delta T}{\sqrt{8}(1-\alpha)^{2}}\left[1+\alpha-\left(\frac{8 \alpha^{2}}{1+\alpha^{2}}\right)^{1 / 2}\right] .
$$




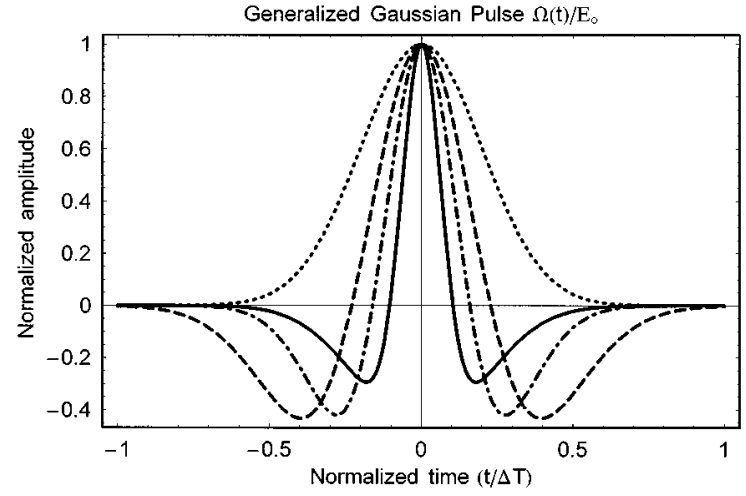

(a)

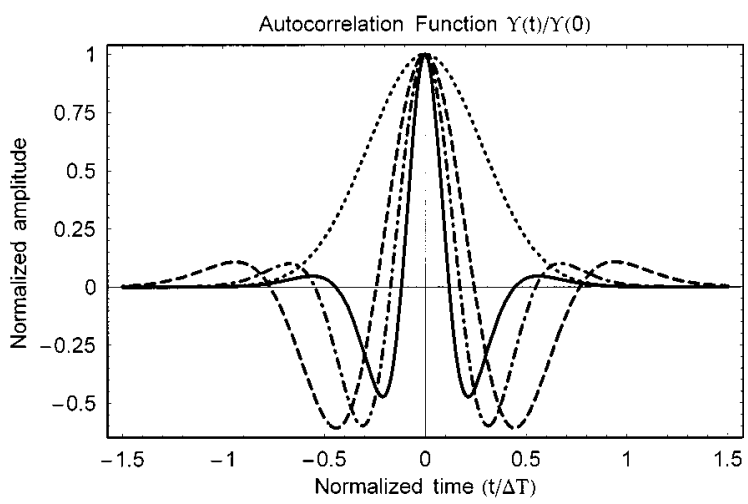

(b)

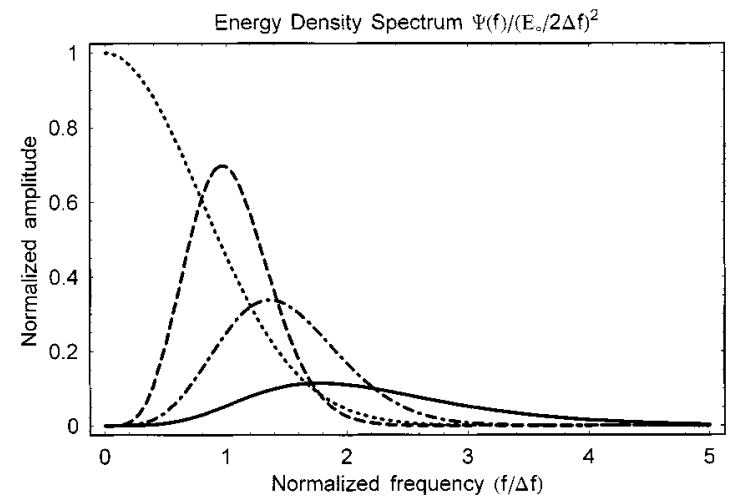

(c)

Fig. 1. (a) Normalized time variation of the generalized Gaussian pulse $\Omega(t) / E_{\circ}$. (b) Autocorrelation function $\Upsilon(t) / \Upsilon(0)$. (c) Energy density spectrum $\Psi(f)=|\Lambda(f)|^{2}$ for values of the scaling parameter $\alpha=0$ (dotted line), $\alpha=0.75$ (dashed line), $\alpha=1.5$ (dashed-dotted line), and $\alpha=3$ (solid line).

The normalized autocorrelation function $\Upsilon(t) / \Upsilon(0)$ is plotted in Fig. 1(b) as a function of relative time $t / \Delta T$ for $t_{\circ}=0$ and $\alpha=0$ (dotted line), 0.75 (dashed line), 1.5 (dashed-dotted line), and 3 (solid line). The autocorrelation function $\Upsilon(t)$ has the same pulse characteristics described above for $\Omega(t)$. In addition, its time variation includes positive time sidelobes of small amplitudes.

The temporal-resolution capability of the GGP signal is defined by the duration of its autocorrelation function $\Upsilon(t)$ at the 3 -dB points (or the half-energy points). According to the plots in Fig. 1(b), the half-energy pulse width is directly proportional to the nominal duration $\Delta T$ and also inversely proportional to the scaling factor $\alpha$. Hence, the temporal resolution $\Delta \tau$ can be expressed as follows:

$$
\Delta \tau=C \Delta T / \alpha=C / \alpha \Delta f
$$

where $C \leq 1$ is a proportionality constant.

The Fourier transform of the signal $\Omega(t)$ results in the amplitude spectral density function $\Lambda(f)$

$$
\begin{aligned}
\Lambda(f)= & \int_{-\infty}^{+\infty} \Omega(t) \exp \{-j 2 \pi f t\} d t \\
= & \frac{E_{\mathrm{o}} \exp \left\{-j 2 \pi t_{\circ}(f / \Delta f)\right\}}{2(1-\alpha) \Delta f} \\
& \quad \times\left(\exp \left\{-(\pi / 4)(f / \Delta f)^{2}\right\}\right. \\
& \left.\quad-\exp \left\{-\left(\pi / 4 \alpha^{2}\right)(f / \Delta f)^{2}\right\}\right)
\end{aligned}
$$

where $\Delta f=1 / \Delta T$ is the nominal frequency bandwidth. The energy spectral density of the signal $\Omega(t)$ is defined as $\Psi(f)=$ $|\Lambda(f)|^{2}$. It is also defined in terms of the Fourier transform of the autocorrelation function $\Upsilon(t)$

$$
\begin{aligned}
\Psi(f) & =\int_{-\infty}^{+\infty} \Upsilon(t) \exp \{-j 2 \pi f t\} d t \\
& =\left(\frac{E_{\circ}}{\Delta f}\right)^{2} \sum_{k=0}^{2} \tilde{I}_{k} \exp \left\{-\tilde{a}_{k}(f / \Delta f)^{2}\right\}
\end{aligned}
$$

where the time constant $t_{0}$ is set equal to zero for simplicity and the coefficients $\tilde{I}_{k}$ and $\tilde{a}_{k}$ are expressed as follows:

$$
\begin{aligned}
\tilde{I}_{k} & =\sqrt{\pi / a_{k}} I_{k} \\
\tilde{I}_{0} & =\tilde{I}_{1}=1 / 4(1-\alpha)^{2}, \quad \tilde{I}_{2}=-2 \tilde{I}_{\circ} \\
\tilde{a}_{k} & =\pi^{2} / a_{k} \\
\tilde{a}_{0} & =\pi / 2, \quad \tilde{a}_{1}=\pi / 2 \alpha^{2}, \quad \tilde{a}_{2}=\pi\left(1+\alpha^{2}\right) / 4 \alpha^{2} .
\end{aligned}
$$

The normalized energy spectral density function $\Psi(f) /\left(E_{\circ} / \Delta f\right)^{2}$ is shown in Fig. 1(c) as a function of relative frequency $f / \Delta f$ for $\alpha=0$ (dotted line), 0.75 (dashed line), 1.5 (dashed-dotted line), and 3 (solid line). For $\alpha=0$, the energy density spectrum $\Psi(f)$ includes dc component at $f / \Delta f=0$, which is the case for the energy density spectrum of an ideal Gaussian pulse. According to Fig. 1(c), increasing the value of the scaling parameter $\alpha$ causes more spreading of the energy density spectrum.

The nominal center frequency $f_{c}$ at which the peak of the energy density spectrum $\Psi(f)$ occurs is defined by the relationship

$$
f_{c}=\frac{\int_{0}^{\infty} f|\Lambda(f)|^{2} d f}{\int_{0}^{\infty}|\Lambda(f)|^{2} d f}=\frac{\int_{0}^{\infty} f \Psi(f) d f}{\int_{0}^{\infty} \Psi(f) d f}
$$

UWB electromagnetic GGP signals with high-energy concentration in the high frequency (HF), very high frequency (VHF), and lower ultrahigh frequency (UHF) region of the spectrum will suffer minimum absorption by lossy propagation media [12], [18]. Hence, the GGP signal has potential uses for the applications of wireless radio communications, ground-penetration radar, and foliage-penetration radar. 


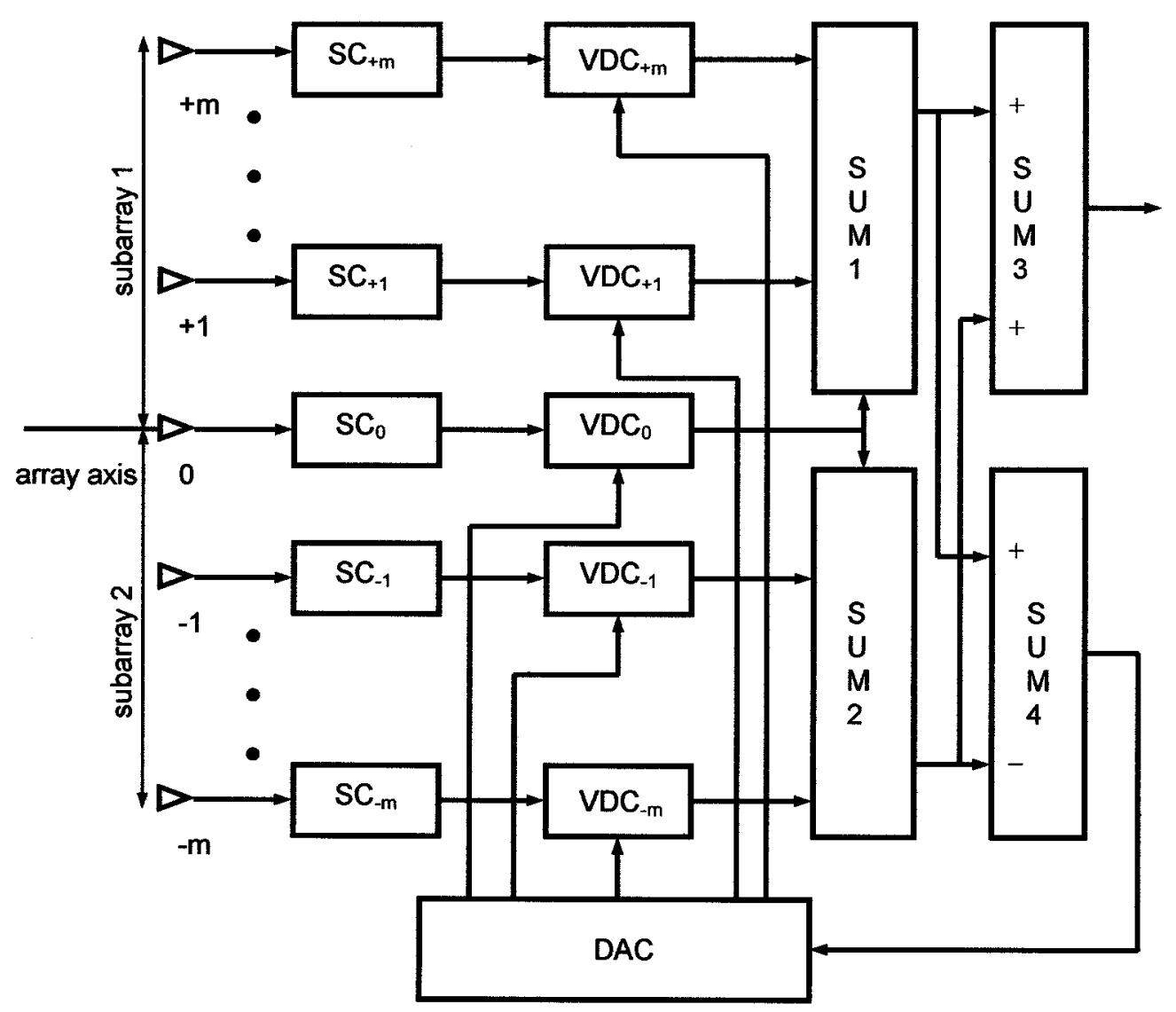

Fig. 2. A self-steering array beamforming system for UWB impulse waveforms. The beamformer includes $2 m+1$ sensors, sliding correlator $\left(\mathrm{SC}_{i}\right)$, variable delay circiut $\left(\mathrm{VDC}_{i}\right)$, delay adjustment computer (DAC), and summer circuits (SUM).

\section{IMPULSE-BASED ARRAY BEAMFORMING}

\section{A. Space-Time Resolution Function}

The principle of array beamforming based on UWB (carrierfree) signals is depicted in Fig. 2. The beamforming system consists of a linear array of $M=2 m+1$ omnidirectional sensors uniformly spaced with interelement distance $d$. Each channel of the beamformer employs a sliding correlator $\left(\mathrm{SC}_{i}\right)$ for temporal processing of the received signal and a variable delay circuit $\left(\mathrm{VDC}_{i}\right)$. The design and operation of correlator and variable-delay circuits for short pulses are described in [24], [25].

The principle of monopulse-tracking radar is applied to the array system in Fig. 2 to determine the angular sector in which the signal source is located. The array sensors are separated into two subarrays of equal size, $L / 2=m d$. The sensors $i=0,+1,+2, \ldots,+m$ form the subarray 1 , and the sensors $i=0,-1,-2, \ldots,-m$ form the subarray2. The output signals from $\mathrm{VDC}_{i}, i=0,+1, \ldots,+m$ are summed by the summer circuit SUM1 while the signals from $\mathrm{VDC}_{i}, i=0,-1, \ldots,-m$ are summed by the SUM2. Furthermore, the output signals from SUM1 and SUM2 are added by the summer circuit SUM3 and their difference is obtained by the summer circuit SUM4. The signal at the output terminal of SUM3 is referred to as the master signal and the difference signal at the output terminal of SUM4 is referred to as the monopulse signal. The monopulse signal conveys to the delay-adjustment computer (DAC) information about the relative angular coordinate of the signal source. The main task of DAC is to control the delay setting of the $\mathrm{VDC}_{i}$ s so that the beam axis of the array system is electronically steered in the direction of the signal source for optimum signal reception.

Let a planar wavefront with the time variation of the GGP signal $\Omega(t)$ given in (3) be received by the array sensors in Fig. 2 . We assume that the signal source is an impulse beacon used in a localizer network or a transmitter emitting voice or data encoded as sequences of UWB electromagnetic impulses. In the case of impulse radar, the signal source is considered as a scattering center. The response of the $i$ th sensor to the received wavefront can be expressed in terms of the voltage signal

$$
\begin{aligned}
v_{i}(t, \phi)= & \Omega\left(t-\tau_{i}(\phi)\right) \\
= & {\left[E_{\circ} /(1-\alpha)\right]\left(\exp \left\{-4 \pi\left[\left(t-\tau_{i}(\phi)\right) / \Delta T\right]^{2}\right\}\right.} \\
& \left.-\alpha \exp \left\{-4 \pi\left[\alpha\left(t-\tau_{i}(\phi)\right) / \Delta T\right]^{2}\right\}\right)
\end{aligned}
$$

where we set the time constant $t_{0}=0$ for simplifying the mathematics. The function $\tau_{i}(\phi)$ in (17) is the progressive time delay between the voltage signals $v_{i}(t, \phi), i= \pm 1, \pm 2, \ldots, \pm m$ and the voltage signal $v_{0}(t, \phi)$ generated at the output of the sensor with the index $i=0$. The relative time delay $\tau_{i}(\phi) / \Delta T$ is a function of the angle of incidence $\phi$ and the distance $d$ between adjacent sensors

$$
\begin{aligned}
\tau_{i}(\phi) / \Delta T & =(i d / c \Delta T) \sin \phi=(i / 2 m) \rho \sin \phi \\
\rho & =2 m d / c \Delta T=L / c \Delta T=L \Delta f / c
\end{aligned}
$$


where $\rho$ is referred to as the spatial frequency bandwidth and $L=2 m d$ is the array length.

Temporal-signal processing is first carried out by the $\mathrm{SC}_{i}$. The voltage signal $v_{i}(t, \phi)$ is correlated in the $\mathrm{SC}_{i}$ with a replica of the GGP signal $\Omega(t)$ to achieve suppression of additive (thermal) noise. The $\mathrm{SC}_{i}$ can also achieve pulse compression for the selective reception of coded waveforms and PN-noise sequences. The response of a correlator circuit is equivalent to that of a "matched filter," which yields the optimum output signal-to-noise ratio. The response of each $\mathrm{SC}_{i}$ is the voltage signal $\Upsilon_{i}(t)=\Upsilon\left(t-\tau_{i}(\phi)\right)=\Omega\left(t-\tau_{i}(\phi)\right) * h_{c}(t)$, where $h_{c}(t)=\Omega\left(T_{0}-t\right)$ is the impulse response of the $\mathrm{SC}_{i}$, $\Upsilon(t)$ is the autocorrelation function given in (4), and the asterisk $*$ denotes time-domain convolution. In the subsequent derivations, we shall set $T_{0}=0$ for simplicity.

In order to achieve electronic beamsteering for enhancing the quality of signal reception from a desired look direction, e.g., $\phi=$ $\phi_{0}$ the variable delay circuit $\mathrm{VDC}_{i}$ applies to the incoming signal $\Upsilon_{i}(t)$ a time delay $\tilde{\tau}_{i}=(i d / c) \sin \phi_{0}$. In analogy to (18), the relative time delay $\tilde{\tau}_{i} / \Delta T$ can also be expressed in terms of $\rho$

$$
\tilde{\tau}_{i} / \Delta T=(i d / c \Delta T) \sin \phi_{\circ}=(i / 2 m) \rho \sin \phi_{\circ}
$$

Finally, the delayed signals $\Upsilon\left(t+\tilde{\tau}_{i}-\tau_{i}(\phi)\right)$ $i=0, \pm 1, \pm 2, \ldots, \pm m$ from the $\mathrm{VDC}_{i} \mathrm{~s}$ are summed by SUM1, SUM2, and SUM3 to produce the beamformer's response function

$$
\begin{aligned}
\tilde{\Upsilon}(t, \phi)= & \sum_{i=-m}^{m} \Upsilon\left(t+\tilde{\tau}_{i}-\tau_{i}(\phi)\right) \\
= & E_{\circ}^{2} \Delta T \sum_{i=-m}^{m} \sum_{k=0}^{2} I_{k} \exp \left\{-a_{k}[(t / \Delta T)\right. \\
& \left.\left.+(i d / c \Delta T)\left(\sin \phi_{\circ}-\sin \phi\right)\right]^{2}\right\} \\
= & E_{\circ}^{2} \Delta T \sum_{i=-m}^{m} \sum_{k=0}^{2} I_{k} \exp \left\{-a_{k}[(t / \Delta T)\right. \\
& \left.\left.+\rho(i / 2 m)\left(\sin \phi_{\circ}-\sin \phi\right)\right]^{2}\right\} .
\end{aligned}
$$

For a large number of array sensors, $m \gg 1$ summation over the index $i$ in (21) can be approximated by integration with the help of the following substitutions:

$$
\eta=i / 2 m, \quad d \eta=d(i / 2 m), \quad i= \pm m \rightarrow \eta= \pm 1 / 2
$$

Multiplying and dividing (21) by the number of sensors $M=$ $2 m+1$ and applying the substitutions in (22) with the approximation $i /(2 m+1) \approx i / 2 m$ for $m \gg 1$ one obtains the integral form of the master signal $\tilde{\Upsilon}(t, \phi)$ expressed as follows:

$$
\begin{aligned}
\tilde{\Upsilon}(t, \phi) & \approx M E_{\circ}^{2} \Delta T \sum_{k=0}^{2} I_{k} \int_{-1 / 2}^{+1 / 2} \\
& \times \exp \left\{-a_{k}\left[(t / \Delta T)+\rho\left(\sin \phi_{\circ}-\sin \phi\right) \eta\right]^{2}\right\} d \eta
\end{aligned}
$$

The integral in (23) can be solved in terms of the generalized error function

$$
\begin{aligned}
\operatorname{Erf}\left[x_{1}, x_{2}\right] & =\operatorname{erf}\left(x_{2}\right)-\operatorname{erf}\left(x_{1}\right) \\
& =\frac{2}{\sqrt{\pi}} \int_{x_{1}}^{x_{2}} \exp \left\{-u^{2}\right\} d u
\end{aligned}
$$

where $\operatorname{erf}(x)=(2 / \sqrt{\pi}) \int_{0}^{x} \exp \left\{-u^{2}\right\} d u$ is the known error function. Let us make the following substitutions:

$$
\begin{aligned}
u & =\sqrt{a_{k}}\left[(t / \Delta T)+\rho\left(\sin \phi_{\circ}-\sin \phi\right) \eta\right] \\
d u & =\sqrt{a_{k}} \rho\left(\sin \phi_{\circ}-\sin \phi\right) d \eta \\
\eta & =-1 / 2 \rightarrow u=\xi_{k}^{-}(t, \phi) \\
& =\sqrt{a_{k}}\left[(t / \Delta T)-\rho\left(\sin \phi_{\circ}-\sin \phi\right) / 2\right] \\
\eta & =+1 / 2 \rightarrow u=\xi_{k}^{+}(t, \phi) \\
& =\sqrt{a_{k}}\left[(t / \Delta T)+\rho\left(\sin \phi_{\circ}-\sin \phi\right) / 2\right] .
\end{aligned}
$$

Insertion of (25)-(28) into the integrand in (23) results in

$$
\begin{aligned}
\tilde{\Upsilon}(t, \phi) \approx & \frac{M E_{\circ}^{2} \Delta T \sqrt{\pi}}{2 \rho\left(\sin \phi_{\circ}-\sin \phi\right)} \\
& \times \sum_{k=0}^{2}\left(I_{k} / \sqrt{a_{k}}\right)\left(\frac{2}{\sqrt{\pi}} \int_{\xi_{k}^{-}(t, \phi)}^{\xi_{k}^{+}(t, \phi)} \exp \left\{-u^{2}\right\} d u\right) \\
= & \frac{M E_{\circ}^{2} \Delta T}{2 \rho\left(\sin \phi_{\circ}-\sin \phi\right)} \\
& \times \sum_{k=0}^{2} \tilde{I}_{k} \operatorname{Erf}\left[\xi_{k}^{-}(t, \phi), \xi_{k}^{+}(t, \phi)\right] .
\end{aligned}
$$

According to (29), for the look direction $\phi=\phi_{0}$ the beamformer in Fig. 2 yields the maximum response signal

$$
\tilde{\Upsilon}\left(t, \phi_{0}\right)=M \Upsilon(t), \quad M=2 m+1, \quad m \gg 1
$$

The normalized two-dimensional function

$$
\Theta(t, \phi)=\left[\frac{\tilde{\Upsilon}(t, \phi)}{\tilde{\Upsilon}\left(0, \phi_{\circ}\right)}\right]^{2}=\left[\frac{\tilde{\Upsilon}(t, \phi)}{M \mathcal{E}}\right]^{2}
$$

is referred to as the space-time resolution function (STRF) of the GGP signal $\Omega(t)$ received by the array beamforming system in Fig. 2. A three-dimensional plot of $\Theta(t, \phi)$ is shown in Fig. 3 as a function of relative time $t / \Delta T$ and angle $\phi$, for (a) $\phi_{0}=0$, $\alpha=3, \rho=10,-5^{\circ} \leq \phi \leq+5^{\circ}$, and (b) $0^{\circ} \leq \phi \leq+3^{\circ}$. The angular-resolution capability of $\Theta(t, \phi)$ is determined by the spacial frequency bandwidth $\rho$ defined in (19) and the scaling factor $\alpha$. The resolution angle is derived in Section III-C. The temporal resolution capability of $\Theta(t, \phi)$ is defined by $\Delta \tau$ given in (9). 


\section{B. Space-Frequency Distribution Function}

The master signal $\tilde{\Upsilon}(t, \phi)$ given in (21) can be expressed in terms of the impulse response function $h_{a}(t, \phi)$ of the array system in Fig. 2

$$
\begin{aligned}
\tilde{\Upsilon}(t, \phi) & =\sum_{i=-m}^{+m} \Upsilon\left(t+\tilde{\tau}_{i}-\tau_{i}(\phi)\right) \\
& =\Upsilon(t) * \sum_{i=-m}^{+m} \delta\left(t+\tilde{\tau}_{i}-\tau_{i}(\phi)\right) \\
& =\Upsilon(t) * h_{a}(t, \phi)
\end{aligned}
$$

where $\delta(t)$ is the Dirac-delta function and

$$
\begin{aligned}
h_{a}(t, \phi) & =\sum_{i=-m}^{+m} \delta\left(t+\tilde{\tau}_{i}-\tau_{i}(\phi)\right) \\
& =\sum_{i=-m}^{+m} \delta\left(t-\Delta T \rho(i / 2 m)\left(\sin \phi_{0}-\sin \phi\right)\right) .
\end{aligned}
$$

For array antenna with innumerable number of elements, $m \gg 1$ the summation in (33) can be approximated by a rectangular pulse of amplitude $A_{a}(\phi)$ and duration $T_{a}(\phi)$. In this case, we write $h_{a}(t, \phi)$ as follows:

$$
\begin{aligned}
h_{a}(t, \phi) & =M \delta(t), & & \text { for } \phi=\phi_{\circ} \\
& =A_{a}(\phi) \Pi\left[t / T_{a}(\phi)\right], & & \text { for } \phi \neq \phi_{\circ}
\end{aligned}
$$

where $\Pi\left[t / T_{a}(\phi)\right]$ is a rectangular pulse of amplitude unity and duration $T_{a}(\phi)$

$$
\begin{aligned}
\Pi\left[t / T_{a}(\phi)\right] & =1, \quad \text { for }-T_{a}(\phi) / 2 \leq t \leq+T_{a}(\phi) / 2 \\
& =0, \text { for elsewhere } \\
A_{a}(\phi) & =M / \Delta T \rho\left|\left(\sin \phi_{\circ}-\sin \phi\right)\right| \\
T_{a}(\phi) & =\Delta T \rho\left|\left(\sin \phi_{\circ}-\sin \phi\right)\right| .
\end{aligned}
$$

According to (36) and (37), the product $A_{a}(\phi) T_{a}(\phi)$ equals the number of sensors $M$ and represents the weight of the array impulse response $h_{a}(t, \phi)$. This number equals the array gain

$$
G=A_{a}(\phi) T_{a}(\phi)=M=2 m+1, \quad m \gg 1 .
$$

The Fourier transform of $h_{a}(t, \phi)$ results in the transfer function or frequency response $H_{a}(f, \phi)$ of the array system in Fig. 2

$$
\begin{aligned}
H_{a}(f, \phi) & =\mathcal{F}\left\{h_{a}(t, \phi)\right\} \\
& =M \operatorname{sinc}\left[\rho\left(\sin \phi_{0}-\sin \phi\right)(f / \Delta f)\right],
\end{aligned}
$$

where $-\pi / 2 \leq \phi \leq+\pi / 2$ the operator $\mathcal{F}\{$.$\} denotes Fourier$ transform and the function $\operatorname{sinc}[x]=\sin (\pi x) / \pi x$. Based on (39) and the convolution relationship in (32), the Fourier trans-

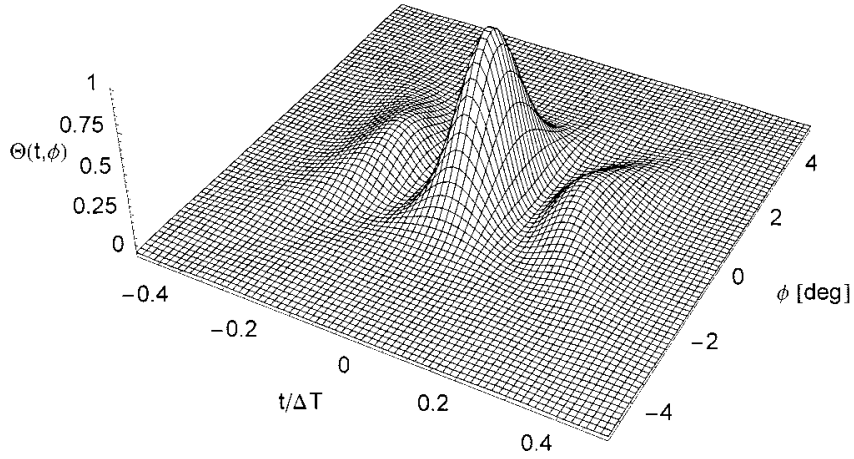

(a)

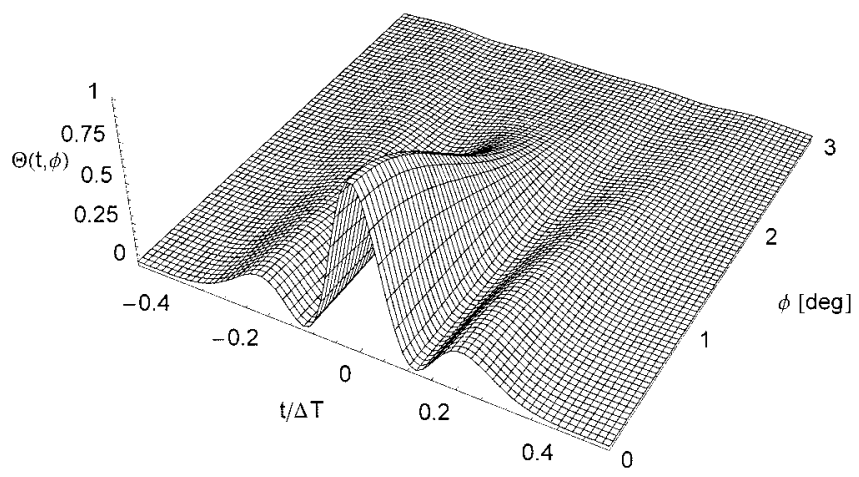

(b)

Fig. 3. Space-time resolution function $\Theta(t, \phi)$ for the value of spacial-frequency bandwidth $\rho=10$. (a) Scaling parameter $\alpha=3$ and the angular range $-5^{\circ} \leq \phi \leq+5^{\circ}$. (b) $0^{\circ} \leq \phi \leq+3^{\circ}$.

form of the master signal $\tilde{\Upsilon}(t, \phi)$ yields the density function

$$
\begin{aligned}
\tilde{\Psi}(f, \phi)= & \mathcal{F}\{\tilde{\Upsilon}(t, \phi)\} \\
= & \mathcal{F}\{\Upsilon(t)\} \times \mathcal{F}\left\{h_{a}(t, \phi)\right\} \\
= & M \Psi(f) \operatorname{sinc}\left[\rho\left(\sin \phi_{0}-\sin \phi\right)(f / \Delta f)\right] \\
= & M\left(E_{\circ} / \Delta f\right)^{2} \operatorname{sinc}\left[\rho\left(\sin \phi_{\circ}-\sin \phi\right)(f / \Delta f)\right] \\
& \times \sum_{k=0}^{2} \tilde{I}_{k} \exp \left\{-\tilde{a}_{k}(f / \Delta f)^{2}\right\} .
\end{aligned}
$$

The normalized energy density spectrum

$$
\begin{aligned}
\Phi(f, \phi)= & {\left[\frac{\tilde{\Psi}(f, \phi)}{\left.M\left(E_{\circ} / \Delta f\right)^{2}\right)}\right]^{2} } \\
= & \left(\operatorname{sinc}\left[\rho\left(\sin \phi_{\circ}-\sin \phi\right)(f / \Delta f)\right]\right)^{2} \\
& \times\left(\sum_{k=0}^{2} \tilde{I}_{k} \exp \left\{-\tilde{a}_{k}(f / \Delta f)^{2}\right\}\right)^{2}
\end{aligned}
$$

is referred to as space frequency distribution function (SFDF). A three-dimensional plot of $\Phi(f, \phi)$ is shown in Fig. 4 for $\phi_{0}=$ $0^{\circ}, \alpha=3$, and $\rho=10$. The plot shows energy distribution over a plane defined by the spacial axis $\phi$ and the relative frequency axis $f / \Delta f$.

\section{Directivity Patterns and Angular Resolution}

According to (29) and the plots in Fig. 3, the peak amplitude of the normalized signal $\tilde{\Upsilon}(t, \phi) / \tilde{\Upsilon}\left(0, \phi_{0}\right)$ is a decreasing 


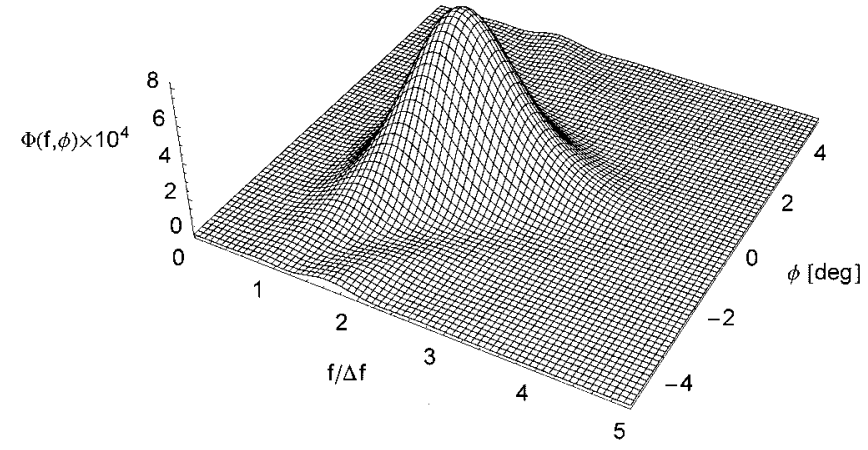

Fig. 4. Space-frequency distribution function $\Phi(f, \phi)$ for the value of spacial-frequency bandwidth $\rho=10$ and scaling parameter $\alpha=3$.

function of the angle of incidence $\phi$. Hence, one can derive a peak-amplitude pattern, also referred to as array factor, for the beamforming system in Fig. 2. The peak-amplitude pattern $\tilde{A}(\phi)$ is defined by the ratio

$$
\tilde{A}(\phi)=\frac{\tilde{\Upsilon}(0, \phi)}{\tilde{\Upsilon}\left(0, \phi_{\circ}\right)}=\frac{\tilde{\Upsilon}(\phi)}{M \mathcal{E}} .
$$

Insertion of (8) and (29) with $t=0$ into (42) results in the peak-amplitude pattern

$$
\begin{array}{rlr}
\tilde{A}(\phi) & =1, & \text { for } \phi=\phi_{0} \\
& =\sum_{k=0}^{2} w_{k} \frac{\operatorname{Erf}\left[\xi_{k}^{-}(\phi), \xi_{k}^{+}(\phi)\right]}{2 \rho\left(\sin \phi_{\circ}-\sin \phi\right)} & \\
& =\sum_{k=0}^{2} w_{k} \frac{\operatorname{erf}\left[\xi_{k}(\phi)\right]}{\rho\left(\sin \phi_{0}-\sin \phi\right)}<1, \quad \text { for } \phi \neq \phi_{\circ}
\end{array}
$$

where the spacial function $\xi_{k}(\phi)$ and the coefficient $w_{k}$ are given by the relationships

$$
\begin{aligned}
w_{0} & =w_{1}=\left[\sqrt{2}\left(1+\alpha-\sqrt{8 \alpha^{2} /\left(1+\alpha^{2}\right)}\right)\right]^{-1} \\
w_{2} & =-2 w_{0} \\
\xi_{k}(\phi) & =\xi_{k}^{+}(0, \phi)=-\xi_{k}^{-}(0, \phi) \\
& =\sqrt{a_{k}} \rho\left(\sin \phi_{0}-\sin \phi\right) / 2 .
\end{aligned}
$$

The identity $\operatorname{Erf}[-x, x]=2 \operatorname{erf}[x]$ is used in (43).

The peak-power pattern $P(\phi)$ is defined as the square of the peak-amplitude pattern

$$
P(\phi)=[\tilde{A}(\phi)]^{2}=\Theta(0, \phi) .
$$

For time-limited (energy) signals, the directivity pattern of the array beamforming system in Fig. 2 can be defined in terms of the ratio of the energy (or average power) $\tilde{U}(\phi)$ received from a given direction $\phi$ to the maximum energy $\tilde{U}\left(\phi_{0}\right)$ received from the direction of the signal origin at $\phi=\phi_{0}$. The signal energy $\tilde{U}(\phi)$ at the output of the beamforming system in Fig. 2 is determined by the following integral:

$$
\tilde{U}(\phi)=\int_{-\infty}^{+\infty}[\tilde{\Upsilon}(t, \phi)]^{2} d t
$$

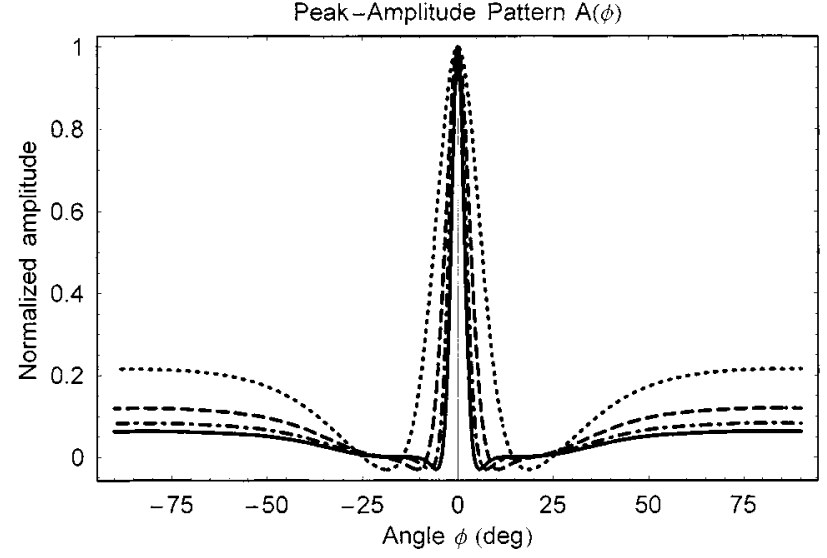

(a)

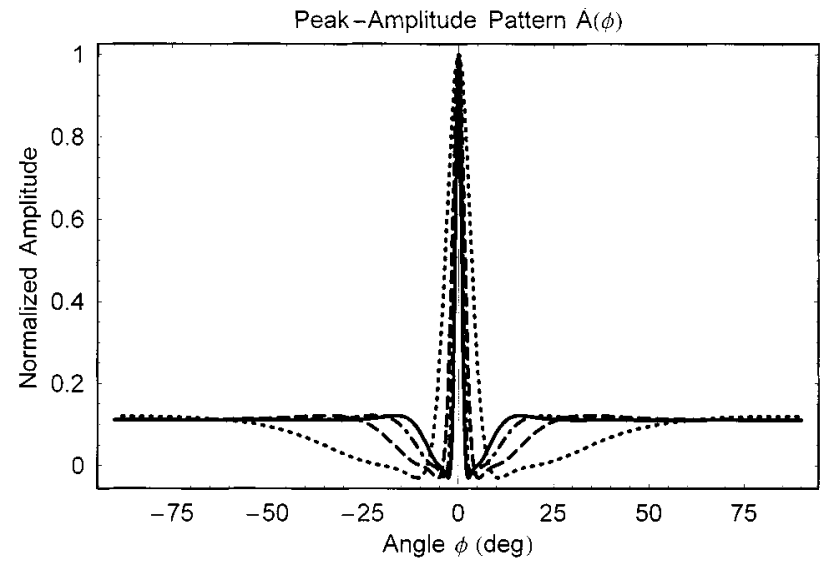

(b)

Fig. 5. Peak-amplitude pattern $\tilde{A}(\phi)$ for: (a) $\phi_{\circ}=0^{\circ}, \alpha=3, d=c \Delta T / 2$, and $M=5$ (dotted line), 9 (dashed line), 13 (dashed-dotted line), and 17 (solid line) and (b) $M=9$ and $d=c \Delta T / 2$ (dotted line), $c \Delta T$ (dashed line), $3 c \Delta T / 2$ (dashed-dotted line), and $2 c \Delta T$ (solid line).

Hence, the directivity energy pattern $\tilde{W}(\phi)$ can be expressed in terms of the ratio

$$
\begin{array}{rlr}
\tilde{W}(\phi) & =\frac{\tilde{U}(\phi)}{\tilde{U}\left(\phi_{0}\right)}=1, & \text { for } \phi=\phi_{\circ} \\
& =\frac{\int_{-\infty}^{\infty}[\tilde{\Upsilon}(t, \phi)]^{2} d t}{\int_{-\infty}^{\infty}\left[\tilde{\Upsilon}\left(t, \phi_{0}\right)\right]^{2} d t}<1, \quad \text { for } \phi \neq \phi_{0} .
\end{array}
$$

The directivity energy pattern can also be expressed in terms of the STRF $\Theta(t, \phi)$ and the SFDF $\Phi(t, \phi)$ as follows:

$$
\tilde{W}(\phi)=\int_{-\infty}^{+\infty} \Theta(t, \phi) d t=\int_{-\infty}^{+\infty} \Phi(f, \phi) d f
$$

Computer plots of the peak-amplitude pattern $\tilde{A}(\phi)$ defined in (42) are shown in Fig. 5. The plots in Fig. 5(a) are based on the response function $\tilde{\Upsilon}(t, \phi)$ given in (21) for the values of scaling factor $\alpha=3$, interelement spacing $d=c \Delta T / 2$, and number of sensors $M=5$ (dotted line), 9 (dashed-line), 13 (dashed-dotted line), and 17 (solid line). The plots in Fig. 5(b) are for the values of $\alpha=3, M=9$, and $d=c \Delta T / 2$ (dotted line), $d=c \Delta T$ (dashed-line), $d=3 c \Delta T / 2$ (dashed-dotted 
line), and $d=2 c \Delta T$ (solid line). According to Fig. 5, the beam pattern $\tilde{A}(\phi)$ drops sharply in the vicinity of the beam axis to an infinitesimal negative value and rises again to rest at a level $1 / M$ for the increasing values of the angle of incidence $\phi$. The rest level for the plots in Fig. 5(a) is $1 / M=0.2$ (dotted line), 0.111 (dashed-line), 0.077 (dashed-dotted line), and 0.059 (solid line). The width of the beam pattern is a decreasing function of the number of sensors $M$ as well as the interelement spacing $d$. The beam pattern does not include any distinguishable sidelobes. The region where the beam pattern $\widetilde{A}(\phi)$ rises from its minimum value to the rest level $1 / M$ is defined as restlobe.

The interelement separation distance $d$ has a significant role in the design of array antenna. In the case of narrow-band sinusoidal waveforms, generally, $d=\lambda / 2$, where $\lambda$ is wavelength. This design restriction prevents the generation of undesirable grating lobes in the (conventional) directivity pattern of array antenna. For UWB carrier-free signals such as the GGP signal, having an effective finite duration $\Delta T$, a separation distance $d \geq c \Delta T / 2$ is of interest, especially if one is restricted by the allowable dimensions of the antenna platform and the number of array elements $M=2 m+1$. According to Fig. 5(b), increasing $d$ does not affect the restlobe level of the beam pattern which is fixed at the value $1 / M=1 / 9=0.111$. The rate at which the restlobe is formed is directly proportional to the interelement distance $d$. Hence, a change in the value of $d$ in proportion to the electric length $c \Delta T$ influences only the beamwidth of $\tilde{A}(\phi)$.

The peak-power pattern $P(\phi)$ and the energy pattern $\tilde{W}(\phi)$ are shown in Figs. 6(a) and 7(a), respectively, for $\phi_{0}=0^{\circ}, \rho=10$, and $\alpha=0.5$ (dotted line), 0.75 (dashed line), 1.5 (dashed-dotted line), and 3 (solid line). The plots are also repeated in Figs. 6(b) and 7(b) for $\alpha=3$ and $\rho=4$ (dotted line), 6 (dashed line), 8 (dashed-dotted line), and 10 (solid line). For $\alpha=0$, (47) and (49) result in the directivity patterns of an ideal Gaussian pulse as shown in Fig. 8 for the different values of $\rho=4,6,8$, and 10. According to Figs. 6-8, the directivity patterns $P(\phi)$ and $\tilde{W}(\phi)$ are sidelobe-free and they are characterized by a mainlobe whose width is a decreasing function of the scaling parameter $\alpha$ and the spacial-frequency bandwidth $\rho$. Hence, larger values of $\alpha$ and $\rho$ result in improved angular resolution and eliminate spacial ambiguities. According to (19), a large value for $\rho$ can be achieved by increasing either the number of array sensors $M=2 m+1$, frequency bandwidth $\Delta f$, or interelement spacing $d$.

The sidelobe-free phenomenon in the directivity patterns $P(\phi)$ and $\tilde{W}(\phi)$ shown in Figs. 6 and 7, respectively, is due to the assumption that the number of array sensors is large $M \gg 1$. This assumption allowed for the derivation of the integral form of the array response $\tilde{\Upsilon}(t, \phi)$ given in (23) and its solution given in (29). For $M \geq 10$ (21) and (29) yield approximately equal directivity patterns withinfinitesimal restlobes; the magnitude of the restlobe for $P(\phi)$ and $\tilde{W}(\phi)$ is fixed at the value $1 / M^{2}$.

The half-power beamwidth of $P(\phi)$ in Fig. 6 and the half-energy beamwidth of $\tilde{W}(\phi)$ in Fig. 7 define the resolution angle for the GGP signal given in (3). According to Fig. 6 through Fig. 8, the beamwidth is inversely proportional to the spacial frequency bandwidth $\rho$ and the scaling factor $\alpha$. Hence, the resolution angle $\Delta \varepsilon$ can be expressed as follows:

$$
\Delta \varepsilon=K / \alpha \rho=K c \Delta T / \alpha L=K c / \alpha L \Delta f
$$

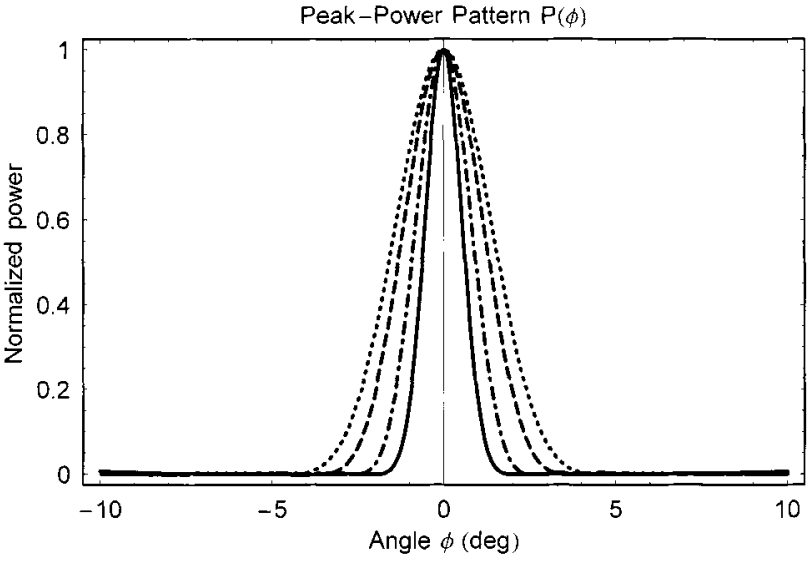

(a)

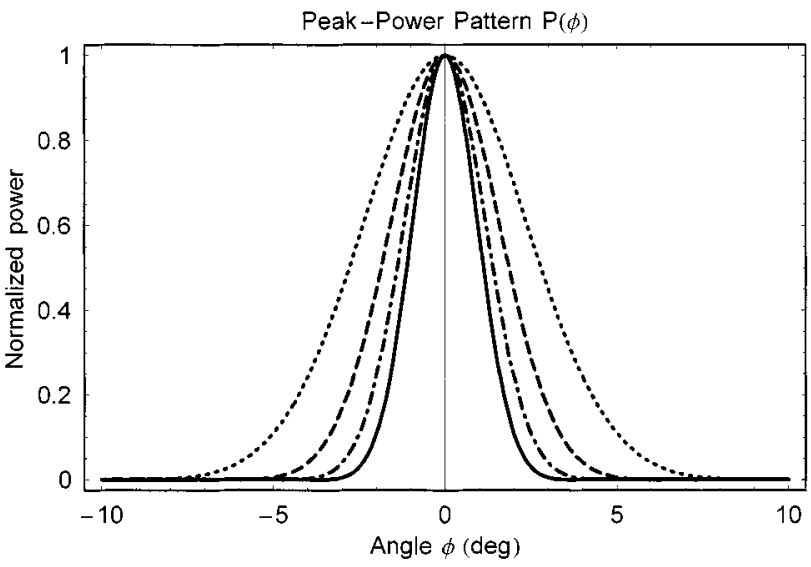

(b)

Fig. 6. Peak-power pattern $P(\phi)$ for: (a) $\phi_{\circ}=0^{\circ}$ and $\rho=10, \alpha=0.5$ (dotted line), 0.75 (dashed line), 1.5 (dashed-dotted line), and 3 (solid line) and (b) $\alpha=3, \rho=4$ (dotted line), 6 (dashed line), 8 (dashed-dotted line), and 10 (solid line).

where $K \leq 1$ is a proportionality constant. The relationships given in (9) and (51) allow for tradeoffs between antenna size $L$, nominal frequency bandwidth $\Delta f=1 / \Delta T$, and scaling parameter $\alpha$ for achieving a combined improvement of temporal resolution as well as angular (or spacial) resolution. This is an advantage in the applications of impulse radar and impulse radio communications for eliminating multipath reception.

For $M=31$ and a pulse duration in the order of $\Delta T=1$ ns, a separation distance $d=c \Delta T=30 \mathrm{~cm}$ results in an array length $L=2 \mathrm{md}=9 \mathrm{~m}$ and the spacial frequency bandwidth $\rho=L / c \Delta T=30$. With these design parameters, one achieves the resolution angle $\Delta \varepsilon=0.033 / \alpha \operatorname{rad}\left(\right.$ or $\left.1.89^{\circ} / \alpha\right)$.

\section{Direction Finding AND EleCtronic BEAMSTEERING}

The sidelobe-free directivity patterns in Figs. 6 and 7 indicate that amplitude weighting is not necessary for array beamforming based on impulse waveforms. It is only necessary to achieve electronic beam steering to optimize signal reception for a specific look direction. The array system in Fig. 2 generates a monopulsetracking signal at the output of SUM4 for the purpose of determining the angular coordinate of the signal source with respect to the beam axis or the array axis in the case $\phi_{0}=0$. This information is required by DAC to adjust the time delay of the $\mathrm{VDC}_{i}$ s for steering the beam axis toward the signal source. 


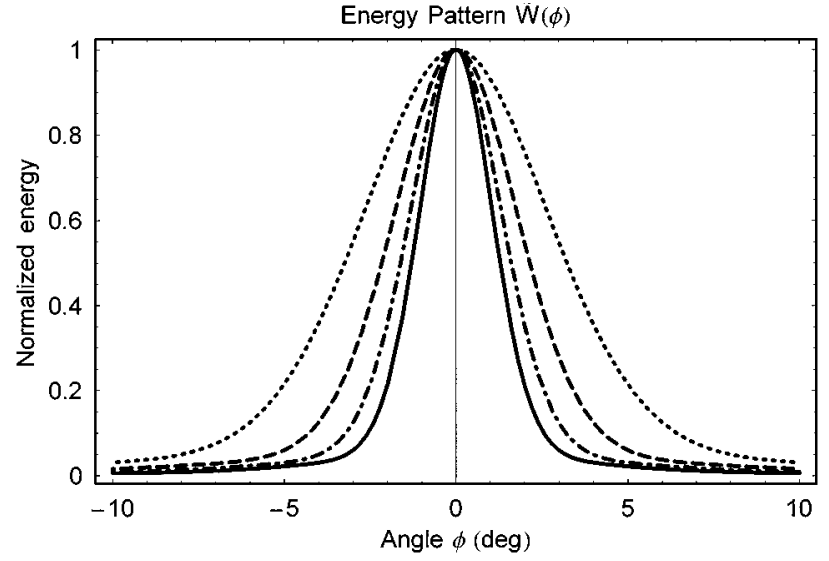

(a)

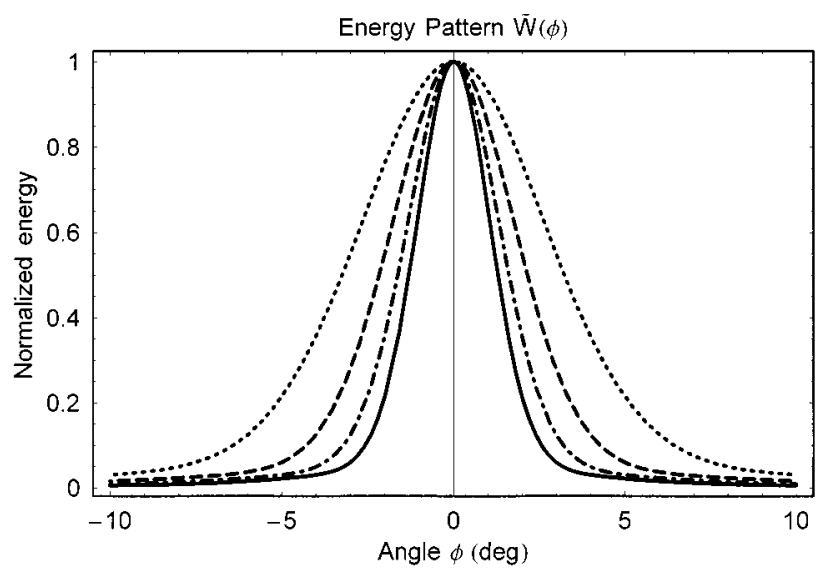

(b)

Fig. 7. Energy pattern $\tilde{W}(\phi)$ for: (a) $\phi_{\circ}=0^{\circ}$ and $\rho=10, \alpha=0.5$ (dotted line), 0.75 (dashed line), 1.5 (dashed-dotted line), and 3 (solid line) and (b) $\alpha=$ $3, \rho=4$ (dotted line), 6 (dashed line), 8 (dashed-dotted line), and 10 (solid line).

\section{A. Monopulse-Tracking Signal}

The monopulse signal delivered to DAC by SUM4 in Fig. 2 can be expressed as follows:

$$
\begin{aligned}
\Upsilon_{\Delta}(t, \phi)=\sum_{i=0}^{+m} \Upsilon\left(t+\tilde{\tau}_{i}-\right. & \left.\tau_{i}(\phi)\right) \\
& -\sum_{i=-m}^{0} \Upsilon\left(t+\tilde{\tau}_{i}-\tau_{i}(\phi)\right) .
\end{aligned}
$$

For $m \gg 1$, the two summations in (52) can be approximated by two integrals. In analogy to (22) and (23), one obtains the approximation

$$
\begin{aligned}
\Upsilon_{\Delta}(t, \phi) \approx & m E_{\circ}^{2} \Delta T\left(\sum _ { k = 0 } ^ { 2 } I _ { k } \int _ { 0 } ^ { 1 } \operatorname { e x p } \left\{-a_{k}[(t / \Delta T)\right.\right. \\
& \left.\left.+\rho\left(\sin \phi_{0}-\sin \phi\right) \eta / 2\right]^{2}\right\} d \eta \\
& -\sum_{k=0}^{2} I_{k} \int_{-1}^{0} \exp \left\{-a_{k}[(t / \Delta T)\right. \\
& \left.\left.\left.+\rho\left(\sin \phi_{\circ}-\sin \phi\right) \eta / 2\right]^{2}\right\} d \eta\right)
\end{aligned}
$$

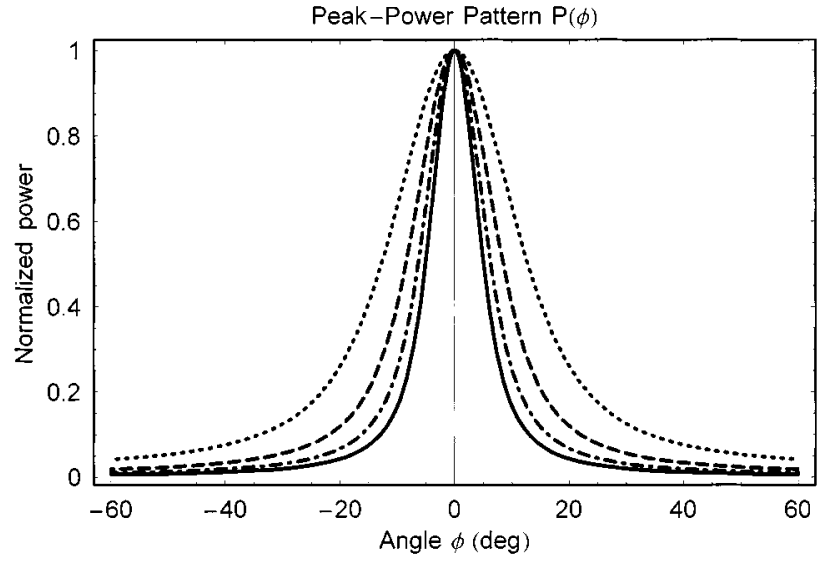

(a)

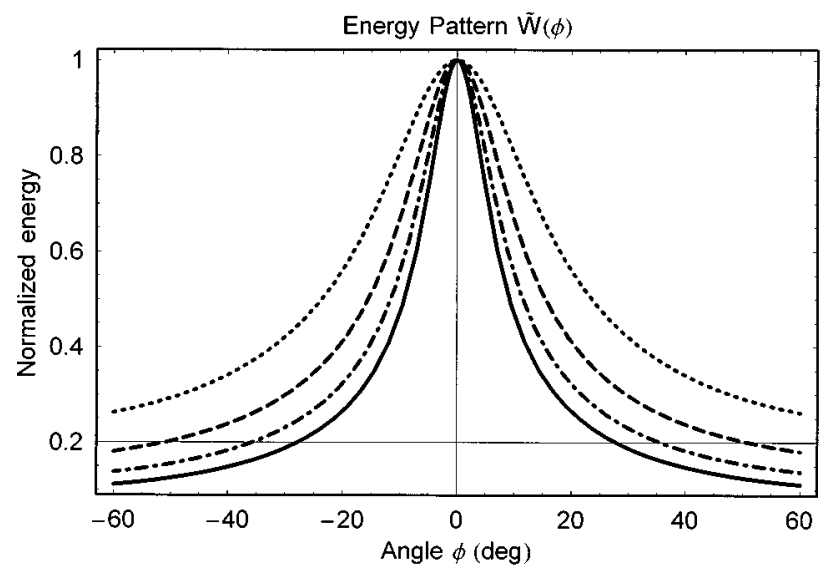

(b)

Fig. 8. (a) Peak-power pattern $P(\phi)$ and (b) energy pattern $\tilde{W}(\phi)$ for the case of an ideal Gaussian pulse with $\alpha=0$. The plots are calculated for $\rho=4$ (dotted line), 6 (dashed line), 8 (dashed-dotted line), and 10 (solid line).

The two integrals in (53) are of the same form as that in (23) and, therefore, they can be solved by considering the following substitutions:

$$
\begin{aligned}
y & =\sqrt{a_{k}}\left[(t / \Delta T)+\rho\left(\sin \phi_{\circ}-\sin \phi\right) \eta / 2\right] \\
d y & =\sqrt{a_{k}} \rho\left(\sin \phi_{\circ}-\sin \phi\right) d \eta / 2 \\
\eta & =0 \rightarrow y=\zeta_{k}(t, 0)=\zeta_{k}(t)=\sqrt{a_{k}}(t / \Delta T) \\
\eta & =+1 \rightarrow y=\zeta_{k}^{+}(t, \phi) \\
& =\sqrt{a_{k}}\left[(t / \Delta T)+\rho\left(\sin \phi_{\circ}-\sin \phi\right) / 2\right] \\
\eta & =-1 \rightarrow y=\zeta_{k}^{-}(t, \phi) \\
& =\sqrt{a_{k}}\left[(t / \Delta T)-\rho\left(\sin \phi_{\circ}-\sin \phi\right) / 2\right] .
\end{aligned}
$$

Inserting (54)-(58) into (53) results in

$$
\begin{aligned}
\Upsilon_{\Delta}(t, \phi) \approx & \frac{m E_{0}^{2} \Delta T \sqrt{\pi}}{\rho\left(\sin \phi_{0}-\sin \phi\right)} \sum_{k=0}^{2}\left(I_{k} / \sqrt{a_{k}}\right) \\
& \times\left(\frac{2}{\sqrt{\pi}} \int_{\zeta_{k}(t)}^{\zeta_{k}^{+}(t, \phi)} \exp \left\{-y^{2}\right\} d y\right. \\
& \left.\quad-\frac{2}{\sqrt{\pi}} \int_{\zeta_{k}^{-}(t, \phi)}^{\zeta_{k}(t)} \exp \left\{-y^{2}\right\} d y\right) .
\end{aligned}
$$




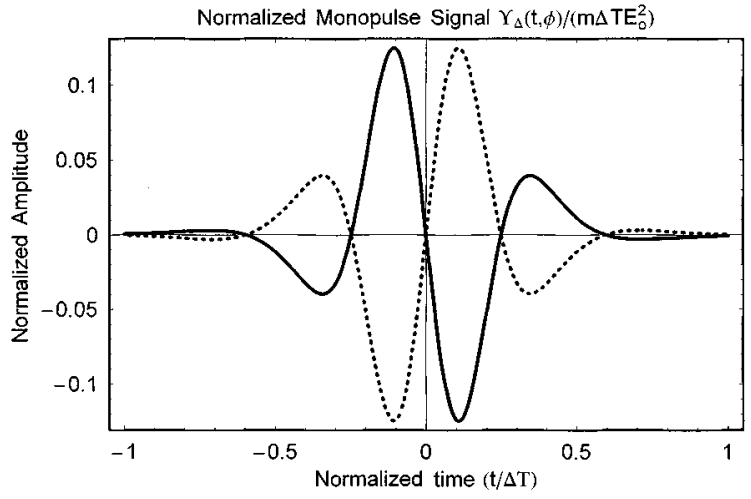

(a)

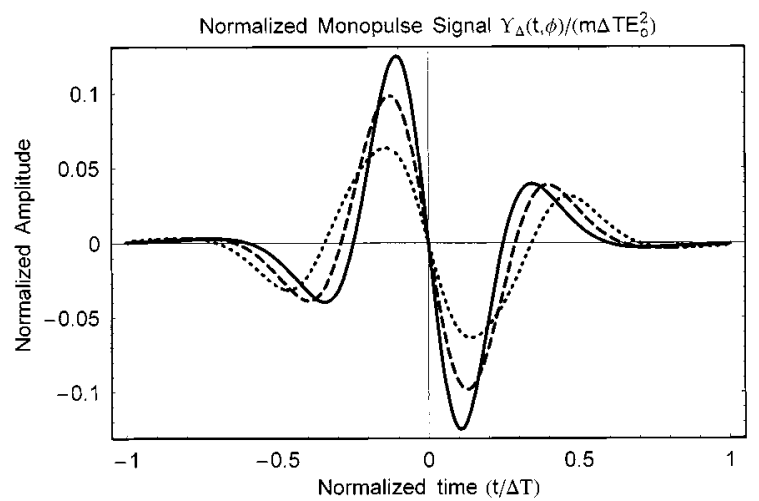

(b)

Fig. 9. Normalized monopulse signal $\Upsilon_{\Delta}(t, \phi) /\left(m \Delta T E_{\circ}^{2}\right)$ plotted as a function of relative time $t / \Delta T$ for: (a) $\alpha=3, \rho=10$, and the values of the angle of incidence $\phi=+2^{\circ}$ (solid line), and $-2^{\circ}$ (dotted line) and repeated for (b) $\phi=2^{\circ}$ (solid line), $3^{\circ}$ (dashed line), and $4^{\circ}$ (dotted line).

According to the definition of the generalized error function given in (24), the two integrals in (59) result in the monopulse signal

$$
\begin{aligned}
& \Upsilon_{\Delta}(t, \phi) \approx \frac{m E_{\circ}^{2} \Delta T}{\rho\left(\sin \phi_{\circ}-\sin \phi\right)} \sum_{k=0}^{2} \tilde{I}_{k} \\
& \quad \times\left(\operatorname{Erf}\left[\zeta_{k}(t), \zeta_{k}^{+}(t, \phi)\right]-\operatorname{Erf}\left[\zeta_{k}^{-}(t, \phi), \zeta_{k}(t)\right]\right) .
\end{aligned}
$$

The normalized monopulse signal $\Upsilon_{\Delta}(t, \phi) /\left(m \Delta T E_{0}^{2}\right)$ is plotted in Fig. 9(a) as a function of relative time $t / \Delta T$ for $\phi_{0}=0^{\circ}, \alpha=3, \rho=10$ and the values of the angle of incidence $\phi=+2^{\circ}$ (solid line), and $\phi=-2^{\circ}$ (dotted line). The same signal is repeated in Fig. 9(b) for the values of $\phi=2^{\circ}$ (solid line), $3^{\circ}$ (dashed line), and $4^{\circ}$ (dotted line).

\section{B. Delay-Adjustment for Beamsteering}

In Fig. 9(a), the monopulse signal for $\phi>0$ is a mirror image of the one for $\phi<0$. Hence, the monopulse signal is characterized by an odd function $\Upsilon_{\Delta}(t, \phi)=-\Upsilon_{\Delta}(-t, \phi)=$ $-\Upsilon_{\Delta}(t,-\phi)$. Based on this odd symmetry, the DAC in the beamforming system of Fig. 2 determines the angular coordinate of the signal source relative to the beam axis at $\phi=\phi_{0}$.

According to Fig. 9(b) and (60), the slope of the line connecting the positive and the negative peak amplitudes of $\Upsilon_{\Delta}(t, \phi)$ is inversely proportional to the angle of incidence $\phi$. Initially, the time-delay $\tilde{\tau}_{i}$ of each $\mathrm{VDC}_{i}$ in Fig. 2 is set to zero by DAC. For on-axis reception, $\phi=0$ the monopulse signal $\Upsilon_{\Delta}(t, 0)=0$ at the output of SUM4 and the master signal $\tilde{\Upsilon}(t, 0)=M \Upsilon(t)$, which is the optimum case for signal reception. For off-axis reception, $\phi=\phi_{0}>0$ the DAC in Fig. 2 receives from SUM4 a monopulse signal with the time variation of the pulse in Fig. 9(a) shown by the solid line and for $\phi=\phi_{\circ}<0$ the dotted line. The DAC calculates an estimate of the angle of arrival $\phi_{0}$ from the slope of the line connecting the positive and the negative main peaks of the monopulse signal. Angle-of-arrival estimation based on slope processing is described in [24]. The delays of the $\mathrm{VDC}_{i} \mathrm{~s}$ are then adjusted by DAC according to the relationship

$$
\begin{array}{ll}
\tilde{\tau}_{i}>\tilde{\tau}_{i+1}, & \text { for }-\pi / 2 \leq \phi<\phi_{\circ} \\
\tilde{\tau}_{i}<\tilde{\tau}_{i+1}, & \text { for } \phi_{\circ}<\phi \leq \pi / 2
\end{array}
$$

where the magnitude of the time delay $\tilde{\tau}_{i}$ is given by (20).

The above delay-adjustment mechanism yields $\Upsilon_{\Delta}\left(t, \phi_{0}\right)=0$ and electronically steers the beam axis of the array in Fig. 2 in the look direction $\phi_{0}$ at which the signal source is located. Any change in the angular position of the source relative to the beam axis will trigger a new monopulse signal that forces DAC to readjust accordingly the delay setting of the $\mathrm{VDC}_{i}$ so that the optimum signal reception is maintained.

\section{CONCLUSION}

The principles of space-time array processing and a mechanism for electronic beam steering are developed based on a realistic signal model for UWB impulse waveforms used in the applications of radar and radio communications. A space-time resolution function and a space-frequency distribution function are derived for impulse waveforms. Impulse-array beamforming yields sidelobe-free directivity peak-power pattern and energy pattern. These beam patterns are characterized by a resolution angle that is a decreasing function of frequency bandwidth and array size. The tradeoff between array size and frequency bandwidth for achieving a high-angular-resolution capability is attractive in practice since signal attenuation can be severe for certain frequency bands. The sidelobe-free feature of the beam patterns is an advantage since it eliminates the need for amplitude weighting at the array elements to achieve sidelobe suppression and provides resistance against multipath reception, cochannel interference, and active jamming.

\section{ACKNOWLEDGMENT}

The author would like to thank Prof. I. Cummings for inviting him to spend sabbatical leave at the Department of Electrical and Computer Engineering, University of British Columbia, Vancouver, BC, Canada, from August 1998 to August 2000. He would also like to thank Profs. R. Word and M. Davies for their support in providing the needed resources to carry out this research work.

\section{REFERENCES}

[1] IEEE Trans. Antennas Propagat. (Spec. Iss. Active and Adaptive Antennas), vol. AP-12, Mar. 1964.

[2] B. Widrow, P. E. Mantey, L. J. Griffiths, and B. B. Goode, "Adaptive antenna systems," Proc. IEEE, vol. 55, pp. 2143-2159, Dec. 1967. 
[3] L. E. Brennan, E. Lawrence, and I. S. Reed, "Theory of adaptive radar," IEEE Trans. Aerosp. Electron. Syst., vol. AES-9, pp. 237-252, Mar. 1973.

[4] R. A. Monzingo and T. W. Miller, Introduction to Adaptive Arrays. New York: Wiley, 1980.

[5] IEEE Trans. Antennas Propagat. (Spec. Iss. Adaptive Processing Antenna Systems), vol. AP-34, Mar. 1986.

[6] B. Widrow and S. D. Stearns, Adaptive Signal Processing. Englewood Cliffs, NJ: Prentice-Hall, 1988.

[7] R. T. Compton, Jr., Adaptive Antennas: Concepts and Performance. Englewood Cliffs, NJ: Prentice-Hall, 1988

[8] W. F. Gabriel, "Adaptive processing array systems, Invited Paper," Proc. IEEE, vol. 80, pp. 152-162, Jan. 1992

[9] M. Barrett and R. Arnott, "Adaptive antennas for mobile communications," Inst. Elect. Eng. Electron. Commun. Eng. J., vol. 8, pp. 203-214, Aug. 1994.

[10] A. J. Paulraj and C. B. Papadias, "Space-time processing for wireless communications," IEEE Signal Processing Mag., vol. 14, pp. 49-83, Nov. 1997.

[11] J. D. Taylor, Ed., Introduction to Ultra-Wideband Radar Systems. Boca Raton, FL: CRC Press, 1995.

[12] H. F. Harmuth, R. N. Boules, and M. G. M. Hussain, Electromagnetic Signals: Reflection, Focusing, Distortion, and Their Practical Applications. New York: Kluwer, 1999.

[13] H. F. Harmuth, Nonsinusoidal Waves for Radar and Radio Communications. New York: Academic, 1981.

[14] M. G. M. Hussain, "Ultra-wideband impulse radar-An overview of principles," IEEE Aerosp. Electron. Syst. Mag., vol. 13, pp. 9-14, Sept. 1998.

[15] M. G. M. Hussain and M. J. Yedlin, "Active-array beamforming for ultra-wideband impulse radar," in Proc. IEEE 2000 Int. Radar Conf., Alexandria, VA, May 2000, pp. 267-272.

[16] S. G. Azevedo and T. E. McEwan, "Micropower impulse radar," in Science and Technology Review. Livermore, CA: Lawrence Livermore Nat. Lab., 1996, (UCRL-52 000-96-1/2), pp. 16-29.

[17] T. E. McEwan, J. D. Kilkenny, and G. Dallum, "World's fastest solidstate digitizer," in Energy and Technology Review. Livermore, CA: Lawrence Livermore Nat. Lab., 1994, (UCRL-52 000-94-4), pp. 1-6.

[18] D. J. Daniels, Surface Penetration Radar. London, U.K.: Inst. Elect. Eng., 1996.

[19] L. Carin, N. Geng, M. McClure, J. Sichina, and L. Nguyen, "Ultrawide-band synthetic aperture radar for mine-field detection," IEEE Antennas Propagat. Mag., vol. 41, pp. 18-33, Feb. 1999.
[20] M. Z. Win and R. A. Scholtz, "Impulse radio: How it works," IEEE Commun. Lett., vol. 2, pp. 36-38, Feb. 1998.

[21] R. A. Scholtz, "Multiple access with time-hopping impulse modulation," in Proc. IEEE Military Commun. Conf. '93 (MILCOM '93), Boston, MA, Oct. 1993, pp. 11-14.

[22] M. G. M. Hussain, "Antenna patterns of nonsinusoidal waves with the time variation of a Gaussian pulse-Part IV," IEEE Trans. Electromagn. Compat., vol. 31, pp. 48-54, Feb. 1989.

[23] _ " "A self-steering array for nonsinusoidal waves based on array impulse response measurement," IEEE Trans. Electromagn. Compat., vol. 30, pp. 154-160, May 1988.

[24] — "Performance analysis and advancement of self-steering arrays for nonsinusoidal waves-I, II," IEEE Trans. Electromagn. Compat., vol. 30, pp. 161-174, May 1988.

[25] S. A. Collins, Jr., B. L. Anderson, and C. Liddle, "True time delay with binary time delay control for large arrays," in Proc. 22nd Annu. Antenna Applications Symp.. Urbana, Sept. 1998.

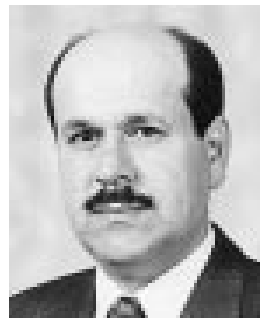

Malek G. M. Hussain (S'80-M'83-SM'90) was born in Kuwait City, Kuwait, in 1953. He received the B.E.E., M.E.E., and Ph.D. degrees in electrical engineering from the Catholic University of America, Washington, DC, in 1977, 1979, and 1983, respectively.

In October 1983, he joined the Department of Electrical and Computer Engineering, Kuwait University, Khaldyah, Kuwait, where he is now a Professor of electrical engineering. From January 1991 to October 1991, he was Dean of Professional and Technological Programs at the Enter-American University, Bayamon, Puerto Rico. From 1991 to 1993, he was the Post-Assistant Vice President for Research and Graduate Studies and from 1993 to 1998 he was Vice President for Academic Support Services at Kuwait University. From 1988 to 1989, he was a Visiting Associate Professor with the Radiation Laboratory, Department of Electrical and Computer Science, University of Michigan, Ann Arbor. From August 1998 to August 2000, he was a Visiting Professor with the Department of Electrical and Computer Engineering, University of British Columbia, Vancouver, Canada.

Dr. Hussain received the 1989 Kuwait Prize for Advancement of Science from the Kuwait Foundation for Advancement of Science. He is a member of the Kuwait Engineers' Society. 